\title{
Towards a Commodity Solution for the Internet of Things
}

\author{
Haifeng Xu ${ }^{a}$, William O. Collinge ${ }^{\mathrm{a}}$, Laura A. Schaefer ${ }^{\mathrm{b}}$, Amy E. Landis ${ }^{\mathrm{c}}$, Melissa M. Bilec $^{\mathrm{a}}$, Alex K. Jones ${ }^{\mathrm{a}}$ \\ ${ }^{a}$ Swanson School of Engineering, University of Pittsburgh, Pittsburgh, PA 15261, USA \\ ${ }^{b}$ Brown School of Engineering, Rice University, Houston, TX 77005, USA \\ ${ }^{c}$ College of Engineering and Science, Clemson University, Clemson, SC 29634, USA
}

\begin{abstract}
Embedded-class processors found in commodity palmtop computers continue to become increasingly capable. Moreover, wireless connectivity in these systems provides new opportunities in designing flexible and smarter wireless sensor networks (WSNs). In this paper we present Lynx, a self-organizing wireless sensor network framework. Leveraging palmtop systems as sensor hubs, Lynx provides fundamental functionality to make a distributively managed, customizable WSN system implementation. Second, we describe Ocelot, a mobile distributed grid-like computing engine for commodity palmtop platforms. The combination of Lynx and Ocelot provides sensor nodes that are capable of collecting, recording, processing, and communicating data without any central server support. Significant energy savings can be achieved for light to medium weight tasks through the Lynx and Ocelot combined system compared to traditional server-class grid-solutions such as BOINC. We demonstrate Lynx and Ocelot in the context of life-cycle building energy usage.

Keywords: Wireless sensor network, self-organizing network, multi-hop routing, distributed computing, palmtop computing, low-power, commodity systems
\end{abstract}

\section{Introduction}

Wireless sensor networks (WSNs) can be broadly defined as any system containing wireless communication and sensing capability. Their applications range from the military domain, including tasks such as battlefield surveillance, to consumer products for in-home energy monitoring and control. As such, WSNs have become an area of significant interest and research activity for a wide variety of academic and commercial research groups. These research topics range from device-level energy harvesting to system-level communication protocol design.

Most of the existing standards for WSNs address specific points in the design space for such systems. The most common standard is the IEEE 802.15.4, more commonly referred to as the ZigBee communication standard. ZigBee is designed to provide inexpensive, low-power, reliable communication over relatively long distances, accomplished in part by utilizing a relatively low data rate in the kbps range. Thus, several WSN products exist that employ the ZigBee communication standard. However, while ZigBee is an attempt 
to optimize the communication protocol for implementing a WSN, WSNs can be constructed using any protocol and hardware platform including common communication standards such as Bluetooth and WiFi (IEEE 802.11).

There is considerable WSN research effort targeting improving system capabilities, including developing algorithms for improving node battery lifetime, system connectivity, and performance $[1,2,3]$. Further, some efforts explore methods for distributing data across the network to maximize data availability when link connectivity is lost, while minimizing system storage and energy overheads $[4,5]$. Goals of this class of research typically involve maximizing WSN lifetime and robustness under a fixed aggregate system energy storage capacity constraint

Most of these efforts work at the algorithmic level and rely on simulation environments to determine their effectiveness. Popular simulators include NetSim [6] and OPNET [7]. Unfortunately, testing new algorithms and ideas in simulation does not always capture the full nature of the system. While certain faults and inefficiencies can be anticipated, use of a real system, even when employing hardware emulation and different physical layer communication protocols, can provide a much more realistic testing environment for WSN proposals.

This paper describes a complete WSN and distributed computing platform that is a first step towards a general commodity solution for nodes in the Internet of Things. Lynx ${ }^{1}$ is a wireless sensor network research environment based on commodity hardware/software systems. Given the proliferation of tablets, smart-phones, and music players, there is a continually expanding platform of devices that can be used to form a WSN. The typical smart-phone or tablet supports multiple communication protocols such as WiFi, Bluetooth, near-field communication (NFC), and GSM. Further, these devices often offer several on-board sensors, including accelerometers, GPS sensors, light sensors, and temperature sensors. These devices are battery operated with relatively long lifetimes, making them highly portable and mobile.

Lynx has two fundamental goals and purposes:

- Allow the creation of a WSN out-of-the-box, leveraging existing sensing and networking capabilities: Using Lynx, it is possible to quickly construct a sensor network from existing devices, many of which are already deployed by users for their more traditional purpose (e.g., smart-phone, tablet function). Lynx can provide access to sensor data from on-board sensors and external sensors.

- Study WSN system advancements using hardware emulation: The relatively low cost, flexi-

\footnotetext{
${ }^{1}$ The University of Pittsburgh mascot is the panther, which in different contexts refers to cougars, jaguars, or leopards. A lynx is a small wild cat related to leopards, and thus, also to panthers. The name was selected for this system because the relationship between a lynx and a leopard is similar to that of a low-power wireless protocol used in WSNs to high-power wireless protocols used in other contexts. Additionally, lynx is a homophone for network "links."
} 
bility, and capability of smart-phones and tablets provides a natural platform for the study of WSN communication and resource management algorithms, including distributed storage and battery maximization techniques. Lynx provides a extensible framework to study these effects in real time and with real environmental conditions.

For integration with Lynx, we present Ocelot $^{2}$, our previous work in the distributed mobile computing area [8]. Ocelot can be used in scenarios where highly parallel, lightweight computational tasks can be partitioned and distributed in an effort to save energy over what would otherwise be required to complete the work with workstation or server machines. An overview of our Lynx and Ocelot system is shown in Figure 1, and discussed in more detail in Section 3. Additionally, Ocelot can be used in an application-specific WSN scenario in which it is difficult/impossible or undesirable to deploy dedicated computational infrastructure. Conceptually, Ocelot is modeled after the Berkeley Open Infrastructure for Network Computing (BOINC) [9], an open source middleware system for volunteer and grid computing on PCs and servers. However, BOINC is complex, requiring considerable computational effort just to initiate and manage connections with the BOINC servers. On a workstation, this overhead is minimal, but on a mobile platform, this overhead is considerable. Ocelot offers a lightweight mechanism to deploy computation tasks on smartphones and tablets using the Android and iOS platforms. Though mobile devices come with limited embedded processing capabilities, their ubiquity can overcome this drawback through their deployment flexibility. Additionally, for lightweight tasks, tablets can often provide much better energy efficiency.

To evaluate the combined Lynx/Ocelot system, we include experiments on executing Ocelot tasks over the Lynx network. This version of Ocelot allows input data to be transferred directly from WSN nodes using a distributed multi-hop routing protocol not requiring a central server as was previously needed for Ocelot [8], allowing operational energy to be reduced by up to $88 \%$.

The remainder of this paper is organized as follows: Section 2 provides some background and description of

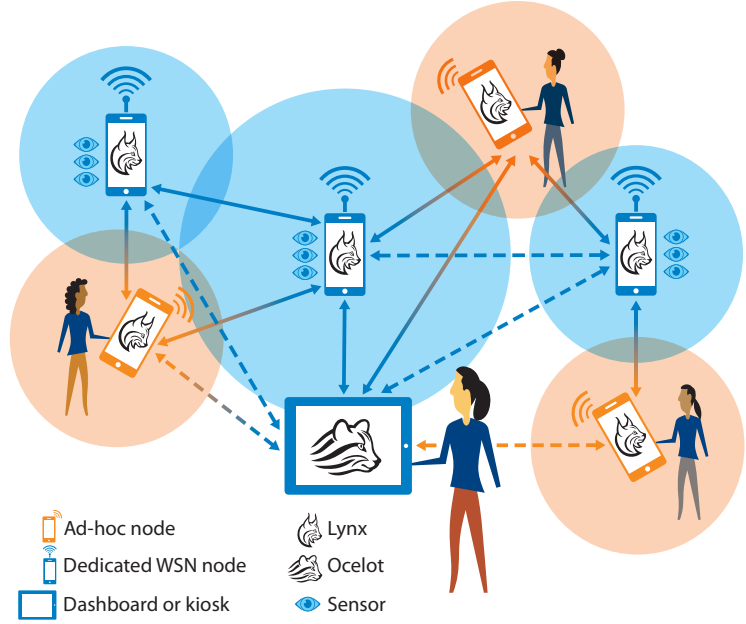
relevant research in WSNs and grid computing. A detailed description of the Lynx approach is contained in

Figure 1: Overview of the Lynx network with the Ocelot distributed computing system.

Section 3. The Ocelot grid computing solution approach is related in Section. 4. Results for using the Lynx

\footnotetext{
${ }^{2}$ Similar to Lynx, Ocelot is also a smaller leopard relative and represents the embedded processor in a tablet in comparison to workstation-class processors.
} 
and Ocelot capabilities on a tablet hardware testbed in a variety of different scenarios and case studies are in included in Section 5. A case study of using Lynx to study different WSN protocols is presented in Section 6 . Section 7 relates conclusions and future work.

\section{Background and Related Work}

In this section, we provide background on wireless sensor networks and grid computing systems, while discussing relevant related work to Ocelot and Lynx in the context of this background.

\subsection{Wireless Sensor Networks and Mobile Connectivity}

The design and construction of wireless sensor networks can be categorized into several aspects including physical layer communication, system-level management, and network organization. In this section, we provide an overview and background on these different layers in the context relevant recent advances in these areas

\subsubsection{Physical Layer Communication}

With the increasing popularity of tablet computers, connectivity technologies and computation capability are rapidly evolving. Commodity wireless protocols such as WiFi and Bluetooth are becoming more competitive with protocols typically designed to work with WSNs such as Zigbee. For example, WiFi 802.11.n with its multiple-input and multiple-output (MIMO) antennas have led to support for new protocols such as WiFi-Direct [10]. WiFi-Direct allows a device to find nearby WiFi-Direct capable devices and form a group to communicate over a peer-to-peer (P2P) link without wireless access points (base stations) in the infrastructure mode. This allows real world implementations of ad-hoc routing to be feasible and robust with commodity devices [11]. Bluetooth Low Energy (BLE) also shows good capability for short range data transmission. It represents a trade-off between energy consumption, latency, size of the Bluetooth connected network or piconet, and throughput [12].

\subsubsection{System-level Management and Optimization}

There have been considerable efforts to improve WSN performance, energy, and battery lifetimes. Relevant research areas include efficient and adaptive ad-hoc routing protocols [1], efficient routing algorithms [2], power management [3], and security [13].

For example, although palmtop devices have significantly larger battery capacities than traditional sensor nodes, the limited energy still remains a major issue in real-world WSN applications. An interesting idea of in-network data aggregation, the TiNA system, has been introduced to save node energy [14]. TiNA 
exploits the temporal correlation in a sequence of sensor readings to suppress values and thus reduces data communication. This concept has been used to investigate sampling and compression at the software [15] and hardware level [16].

\subsubsection{Self-Organizing Network}

The Self-Organizing Network (SON) was originally proposed for the Long Term Evolution (LTE) mobile technology [17]. SON configures and optimizes the increasingly large and complex mobile networks automatically in order to reduce manual interactions and improve network efficiency. A SON generally features functionality such as self-configuration, self-optimization, and self-healing [18]. Thus, adding or dropping network nodes should be managed automatically while maintaining a dynamic load balance. When failures occur, the network structure must also be reconfigured to reduce negative impacts.

The initial focus of SON development was to reduce operating costs in mobile radio networks [18], but the concept is now increasingly recognized as a method for any network to intelligently organize and manage its nodes at scale. The challenges of organizing a peer-to-peer network on a small number of wireless sensors include limited radio range and high geographical volatility. This suggests the need for an adaptive and efficient self-organizing solution such as the Automatic Neighbor Relation (ANR) detection feature found in a SON. Thus, nodes within the radio range of each other can communicate directly; nodes out of range are still able to communicate with the help of one or more intermediate bridge nodes.

\subsection{BOINC, Ocelot, and Distributed Computing}

BOINC is a middleware system connecting central servers and numerous distributed client computers for public-resource computing and storage in areas as diverse as mathematics, medicine, molecular biology, climatology, and astrophysics. BOINC makes it possible to aggregate the enormous processing power of the public volunteered personal computers $(\mathrm{PCs})$ all around the world. Ocelot uses principles similar to BOINC focused on moving lightweight tasks from less power efficient workstations onto ubiquitous palmtop computers [8]. This allows the use of the newly pervasive resource of tablet computers with reduced energy per computation compared to traditional servers.

To our best knowledge, there is no existing system similar to Lynx and Ocelot, which utilizes commodity mobile devices while providing distributed computation capability, without any particular node-level technology requirements such as Zigbee protocol. The extensive work on algorithms and policies to optimize route finding, energy consumption, and security are all orthogonal to and can be applied in our system. Our system can replace or augment software-based simulators to examine newly developed WSN ideas as introduced in Section 1. 


\section{Lynx: A Self-Organizing WSN (SOWSN) Platform Leveraging Commodity Hardware}

Figure 1 provides an overview of how Lynx can construct a WSN from commodity tablets. A dedicated WSN node (fixed and pre-configured) consists of the palmtop computer running the Lynx client with one or more sensors monitoring certain physical or environmental conditions. Sensor data may be stored locally and/or passed through wired (e.g. USB) or wireless connections (e.g. WiFi or Bluetooth) on the Lynx node. Transient mobile devices (such as a smart-phone sitting in

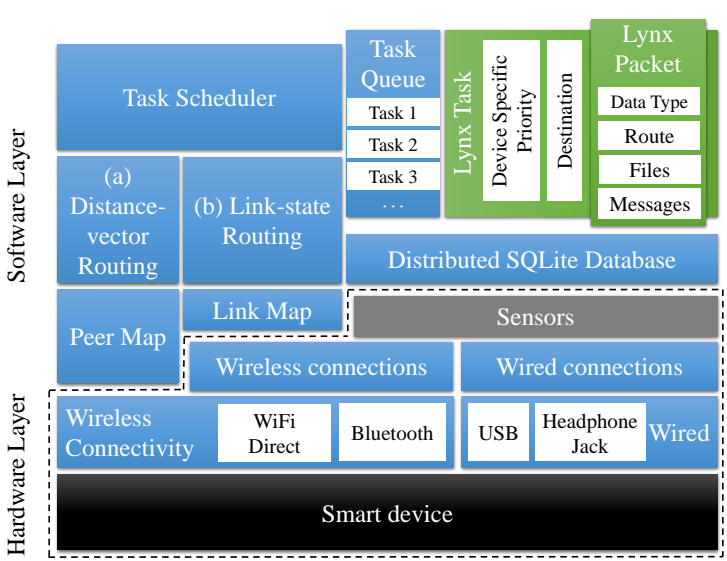

Figure 2: The structure of a Lynx node a pocket) may run the Lynx software and become an ad-hoc Lynx node. Though not required to gather sensor data, ad hoc nodes can serve as a bridge and help to relay data within their connection coverage and capacity. Figure 2 demonstrates how Lynx node modules self-organize and their relationship. Details regarding different module functions are explained in the remainder of this section.

\subsection{Lynx Basics}

There are three elements in Lynx: a Lynx node, Lynx packet, and Lynx task. A Lynx node integrates all useful information related to the local node such as peer map, link map, task queue, etc., as well as relevant methods for handling the information. A Lynx packet is a data wrapper of messages or files, including the packet data type, a packet route, and the packet content type. A Lynx task is a subclass of Lynx packet, with additional fields like task type, next hop address, and the packet payload. Lynx tasks are generated and pushed into a task queue in the Lynx node, which is checked periodically.

\subsection{Connectivity Choice}

The choice of network protocol is independent of the Lynx implementation. In our implementation we have demonstrated the self-organizing concept with both WiFi-Direct and Bluetooth. WiFi-Direct offers advantages because of its wider coverage and better speed. Unfortunately, due to fundamental limits in the network stack, it was only possible for a maximum of two devices to be connected in pairs simultaneously. Changing connection pairs required unacceptably high overhead (e.g. seconds), which made multi-hop routing unstable and inefficient. Given that WiFi-Direct is relatively new, the qualities of driver and application programming interfaces (APIs) still have room to improve. Fortunately, connectivity protocols can be abstracted and all functional modules can be developed upon that abstraction layer [19]. We separated high level design APIs with low level physical layer interfaces in Lynx. Thus, we demonstrated the system using 
Bluetooth connectivity for the current version of Lynx app on Android. As other better link protocols are developed and become mature, Lynx will be able to leverage them, even in a hybrid, multi-protocol fashion.

\subsection{Sensor Integration}

Reading, recording, and sending sensor data are the most important capabilities of a WSN node. Several prevailing commodity mobile platforms, such as iOS and Android, provide hardware capable of doing those tasks. Wireless protocols such as WiFi, Bluetooth, or NFC can be used to connect mobile devices to sensors. It is also possible to send analog sensor data through the $3.5 \mathrm{~mm}$ headphone jack by modulating and demodulating the analog signals [20]. Sensed data can be stored both in files or as database entries on the device. By querying the node connected with the sensor, other nodes in the network can retrieve the sensor data in real time over the established P2P network. We demonstrated Lynx with a Bluetooth enabled Sensordrone containing nine unique environmental sensors. The Lynx node established a point-topoint connection to the Sensordrone and made the sensor data available remotely either through a direct or multi-hop link as discussed in Section 3.5.

\subsection{Maintenance of Peer and Link States}

Wireless APIs typically provide functions such as discovering nearby devices, remembering paired devices, connecting to/disconnect from a remote device, listening to a server socket for incoming connections, etc. A Bluetooth MAC address is a unique string that identifies a device, and can be used to initiate connections to that device. There is a 128-bit universally unique identifier (UUID) that serves to identify radio frequency communication (RFCOMM) channels during the connection establishment. After two devices are connected via Bluetooth, additional sockets can be created to transfer data.

Conceptually, a similar process works for other relevant wireless protocols, including WiFi-Direct. To achieve feasible and efficient routing, each in-network node must have a realtime, global knowledge of the current network structure. MAC addresses are used to uniquely identify devices. Since each device knows its

\begin{tabular}{|c|c|c|c|}
\hline 10:BF:48:CC:79:8C & 50:46:5D:C1:52:3E & 10:BF:48:D0:08:CA & \\
\hline 50:46:5D:C1:52:3E & 10:BF:48:CC:79:8C & & \\
\hline 10:BF:48:D0:08:CA & 10:BF:48:CC:79:8C & 30:85:A9:44:DB:3A & 70:F9:27:76:08:C9 \\
\hline 30:85:A9:44:DB:3A & 10:BF:48:D0:08:CA & & \\
\hline 70:F9:27:76:08:C9 & 10:BF:48:D0:08:CA & & \\
\hline
\end{tabular}

Figure 3: A Lynx peer map illustration. MAC addresses are used in the Lynx network to uniquely identify devices. nearby devices, they can tell others their peer lists (a list of MAC addresses), and at the same time receive and record others' peer lists. Peer lists form a peer map as illustrated in Figure 3. Similarly, a link list and a link map are also maintained, indicating the connection status of local device and the whole network, respectively. To maintain the two lists and two maps, Lynx provides the following functions:

- Peer list scan: periodically scans nearby nodes and updates the peer list. 
- Peer connect: periodically connects to Lynx nodes in its peer list but not in its link list, aborts if number of failures exceeds a threshold.

- Peer list report: after each scan, broadcasts its peer list changes (if any) to connected neighbors.

- Link state report: broadcasts its connection status changes (if any) to connected neighbors.

- Peer/link map report: periodically broadcasts its peer/link map to connected neighbors.

- Task checker: periodically checks if there are any available Lynx tasks in the local task queue.

- In-network sensor connection status: broadcasts sensor connect/disconnect events and connection information to all nodes in the same network (using multi-hop routing if necessary). Information includes the sensor name, MAC address, connected node name, and node address.

\subsection{Multi-hop Routing}

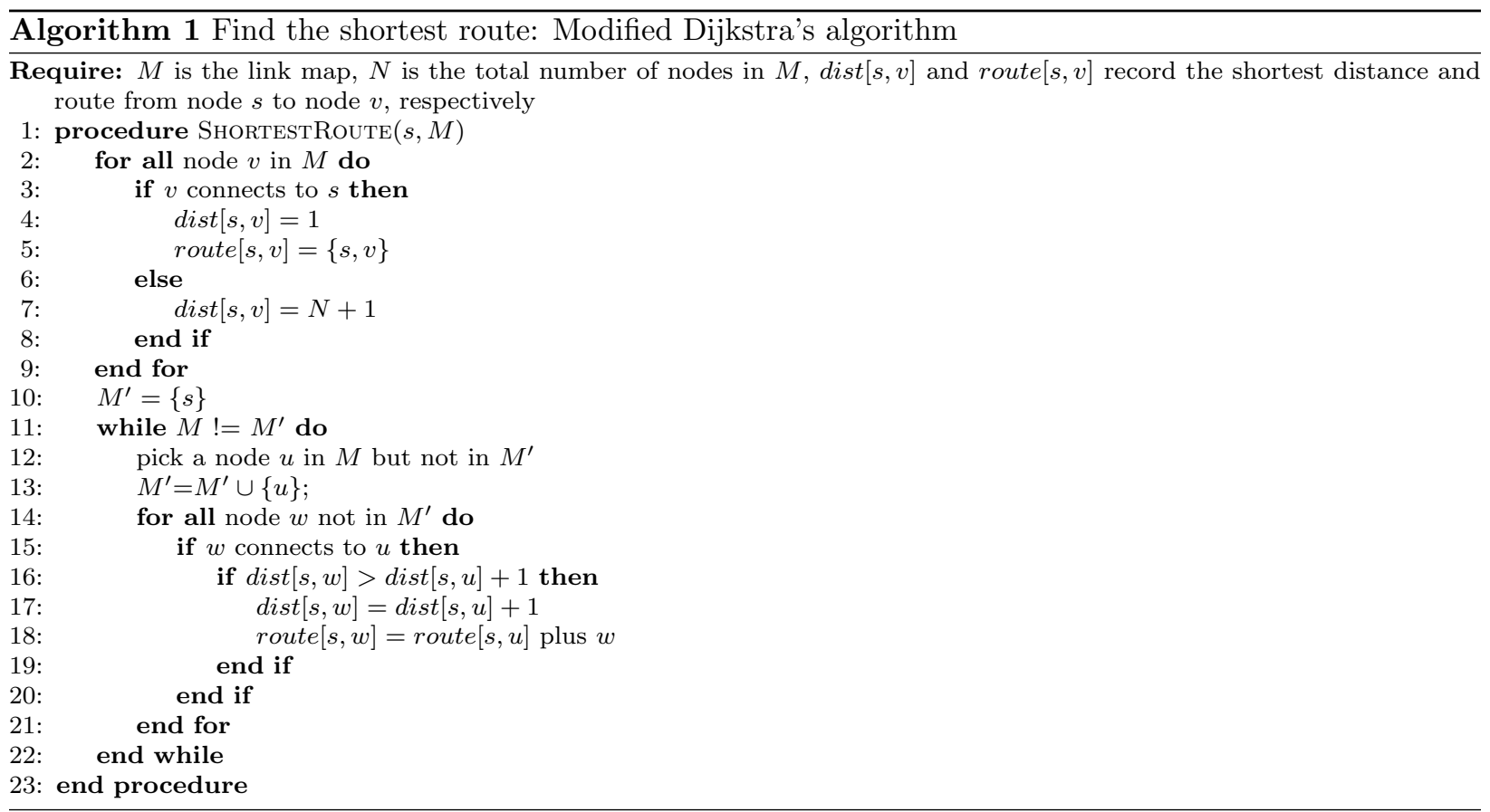

When two nodes are not directly linked but need to communicate, it is necessary to find an indirect route through appropriate intermediate nodes to establish the connection, as shown with dashed lines in Figure 1. From our global link map, we can find the optimal route by modifying some classical link-state routing algorithms, such as a modified Dijkstra's algorithm (Algorithm 1). If the distance of each connected node pair is set to one, the shortest route represents the route with fewest hops, but this approach can be extended to consider other factors such as link quality or remaining energy of different nodes. 
A 4-hop message routing test is shown in Figure 4. Four Android tablets were connected only to adjacent devices as shown in each tablet's "Connected Nodes" list. "SONTester0" (hop 0) was tasked to send a message to "Nexus 7" (hop 3). Lynx calculated the best route (in this case, the only feasible route, SONTester0 $\rightarrow$ SONTester $1 \rightarrow$ SONTester $2 \rightarrow$ Nexus 7 ) and sent the message to the destination via two bridges in between, as shown in the MESSAGE record of the app window. The specific routing algorithm is implemented only to show the feasibility of the Lynx WSN infrastructure to conduct experiments. Lynx, as a platform, has the flexibility to incorporate any routing algorithms, either Link-state or Distance-vector, as illustrated in Figure 2 (a),(b), as well as those optimized for other criteria such as minimizing energy, avoiding nodes with a low battery, etc.

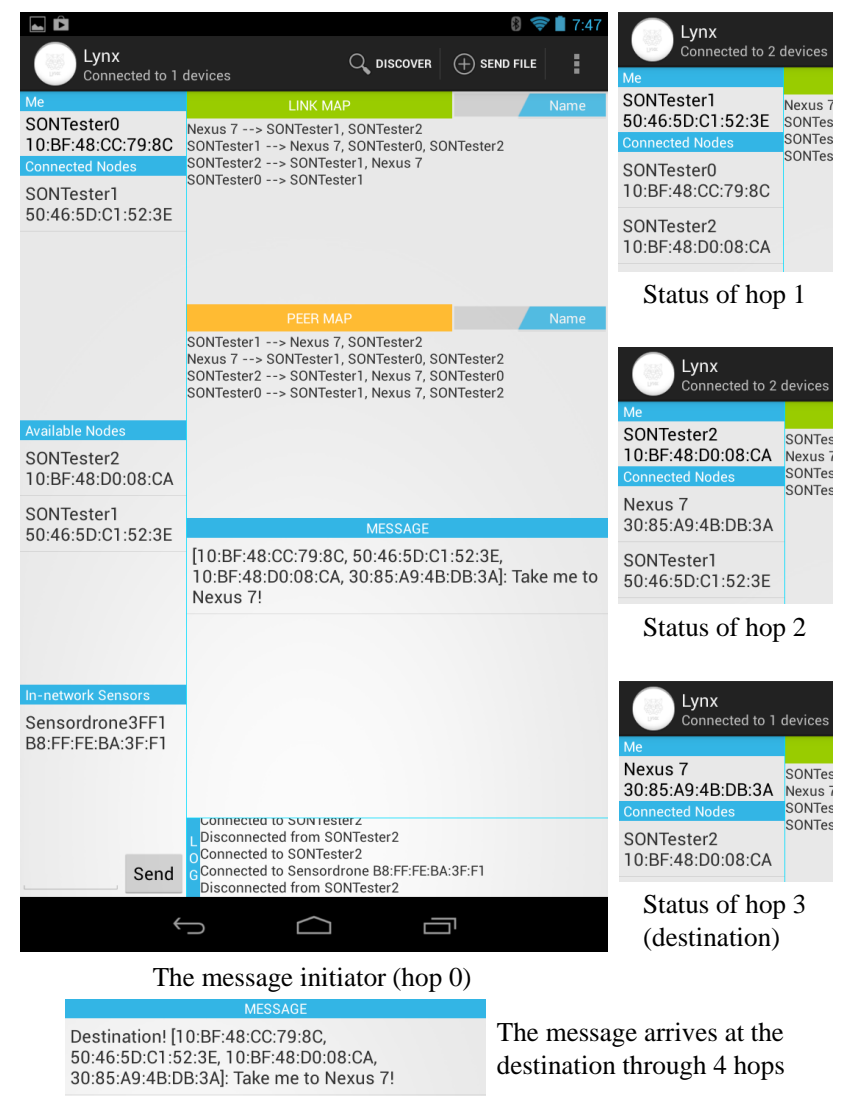

Figure 4: Sending a multi-hop message

\subsection{Scalability and Limitations}

Theoretically, there is no limit for the scale of the network, although performance can degrade as tasks like finding the shortest route increase in runtime when the size of the link map grows. For example, the runtime of our modified Dijkstra algorithm increases by $O(n \log n)$ in the size of the system. However, in practice, the time to finding a route tends to be negligible compared to the data transfer time. When the density of the network grows due to the throughput limit of a single node, transmission rate of each individual route going through the same node will reduce. However, even for a system with over 100 nodes, the network density remains reasonable. Thus, in conclusion, the Lynx network is capable of scaling while maintaining reasonable performance in real world applications.

\section{Joining the Ocelot Compute Engine with Lynx}

When constructing a WSN from commodity tablets, the processing power of sensor nodes remains capable of taking on moderately complex computational tasks with high energy efficiency. With large numbers of these devices connected, computationally complex algorithms such as signal and image processing tasks on 
sensor data can be effectively completed directly by the WSN through task partitioning onto independent nodes' processors.

Ocelot provides a grid-like (or gridlite) computing environment designed specifically for tablet computing environments [8]. Figure 5 shows an overview of the Ocelot system. The benefits of leveraging the embedded processors found in tablets are reduced cost, reduced heat, and lower power usage. Ocelot provides a mechanism to explore and utilize the processing power

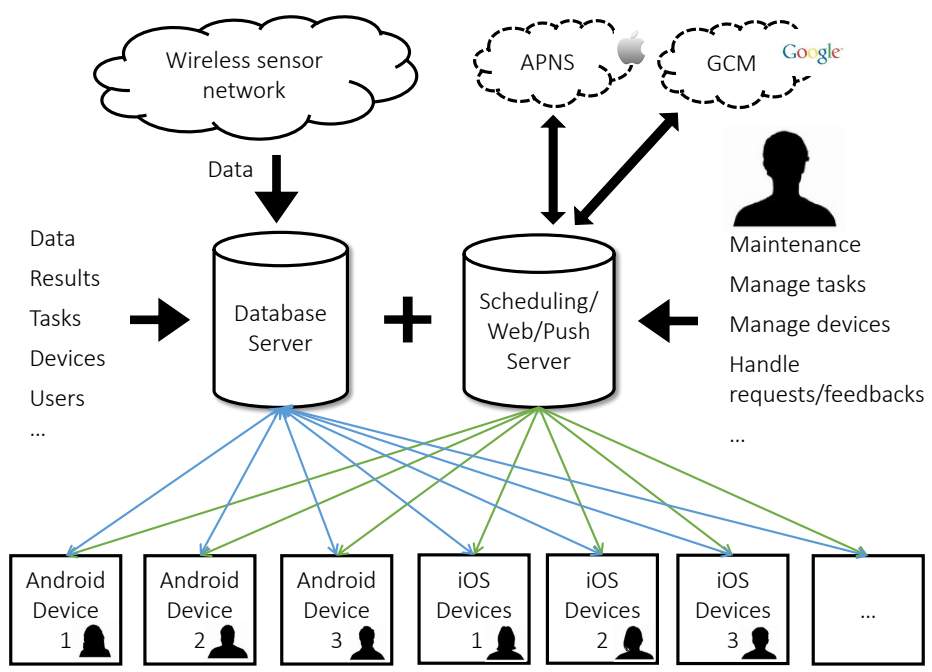

Figure 5: Overview of the Ocelot system within those palmtop devices while balancing their aggregate energy storage according to rules specified by the user. By partitioning tasks and assigning them to tablet devices in parallel, significant speedups can be obtained while maintaining a lower energy consumption than traditional computers.

Lynx provides a natural complement for Ocelot. Prior to Lynx, Ocelot mobile clients were required to be connected to and obtain input data from a central server to complete its computation. Upon task completion, it would upload the results back to server. Though Ocelot utilized the computational power of mobile devices, their mobility and flexibility was not well exploited. Lynx provides a distributed, selforganizing, peer-to-peer network in which data communication can also happen distributively. Thus, Ocelot can be used on a system governed by a Lynx network without requiring central server support. In the reminder of this section, we provide a detailed explanation of Ocelot system.

\subsection{Ocelot Basics}

Similar to BOINC, each Ocelot project is associated with a master URL, which traditionally became the home page address of the project website. Thus, a script or file address under the website directory contains the master URL string as its prefix. We call a data set a workunit, and a compute function an application. A workunit is the smallest unit of input/result data into which the computational task can be divided. Extensible Markup Language (XML) was used as the data wrapper format in Ocelot. XML's generality in representing arbitrary data structures helps the server, the client, and a display/dashboard to generate, communicate, and identify data information in a well-organized fashion.

Input and result workunit examples are shown in Listing 1 and 2, respectively. An input/result XML file may contain more than one workunit and the size is decided according to the computation effort when 
generating the task. A task is the smallest partitioned unit of work to be completed, and consists of a unique task id, creation time, project, application, application version, input file download address, result file upload address, current status, and last status update time. The upload process not only stores the result file to the requester, but also parses its contents and records them in a project table. After a task is finished by one Ocelot client, the result file address is also added to that information entry along with the account name of the contributor, usually the client device's owner. Ocelot provides a simple version of credits for completing a number of tasks.

An Ocelot project can be partitioned into units of work to be completed. These units are called applications, and become tasks within the Ocelot environment. Applications consist of segments of executable code that can be launched by a client on a dataset from the requester and require processing by that project. The code can be stored in different forms for different platforms, and can either be integrated in the client program or later downloaded to the client. Details are explained in Section 4.3. Tailoring the application code is the most important part of efficiently using Ocelot. Ocelot nodes can, in many cases, execute interpreted (e.g., Java) or compiled (e.g., C) codes. Further, they can often utilize multi-threading, and potentially graphics-processing acceleration, but require tight memory management for efficient operation.

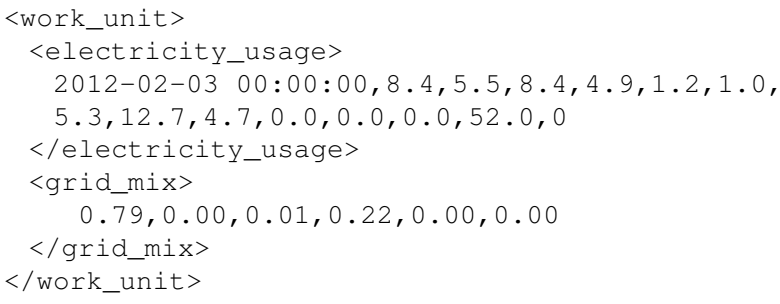

Listing 1: An input workunit example.

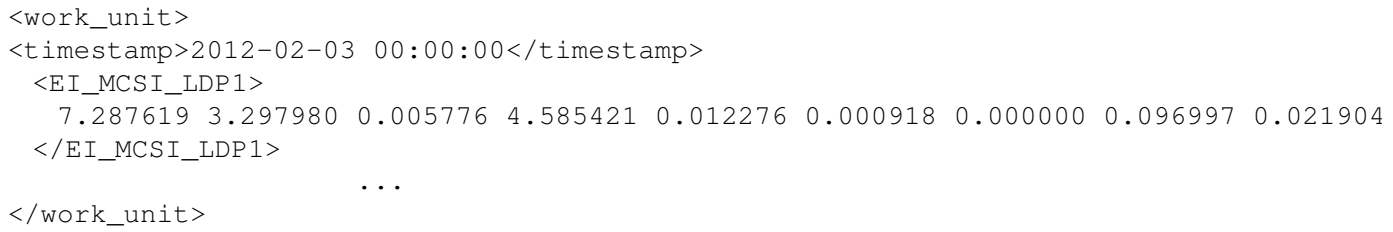

Listing 2: A result workunit example.

By separating applications with data sets, project owners gain more flexibility in adjusting task loads and assigning work from different applications simultaneously. Potential optimization or additional functions can also be added to the application by simply releasing a new version instead of modifying the whole task.

\subsection{Ocelot Servers}

As shown in Figure 5, Ocelot servers store data, schedule computation tasks, communicate with Ocelot clients, and provide interactive functions to both end users and system administrators. Currently, the Ocelot 
server runs on a computing workstation ${ }^{3}$. The Ocelot Dashboard provides a tablet-based interface to the Ocelot server.

The database server is a core component of an Ocelot project. All project related information, including raw data and results, is stored in the database. Additional scheduling information, including task descriptions, accounts, and registered devices, is also stored in the database. The scheduling server handles task requests from clients, generates new tasks from unattended data, and distributes tasks according to the device status. Details about the device status are explained in Section 4.3. The push server utilizes push notification APIs from services like Apple and Google to communicate with iOS and Android devices, respectively. The push service in Ocelot makes bidirectional communication possible and efficient by avoiding the need for the clients to poll the server for work at particular time intervals. Instead, task assignment or other remote operations on clients can happen immediately by pushing pre-defined messages reducing the consumption of battery life in clients. A private push module using P2P links instead of commercial servers was developed for the integration of Ocelot with Lynx.

We provide a brief overview of the main functions in Ocelot:

- register a mobile client node as available for use in the system, based on the client policy selected by the user.

- unregister the client from operation in the system. Systems that become idle for long periods of time (e.g., out of range, exhausted battery, etc.) are automatically unregistered. They must re-register when they become available.

- battStat reports the remaining battery life in a client, and its charging state.

- dataLookup is used to look up raw data, pre-processed input data, and computed results according to specified criteria such as date, whether or when the data was processed, etc.

- deviceLookup lists information about devices registered with the Ocelot server. Each entry includes a unique device id, the platform, owner's account, power connection status, remaining battery, etc. Regularly updated information is needed to calculate aggregate battery and computing power available.

- taskLookup lists information of both active and completed tasks. Active task entries include: id, project/application, creation time, last update time, compute status. Completed task entries additionally include finish time, contributing device id and owner's account, result location, etc.

- fileDownload accesses application code or input data files from a server.

- resultUpload submits computed results to a server.

- taskFeedback is called when a task is finished, migrating it to the finished task table.

\footnotetext{
${ }^{3}$ Many of the server functions have been migrated to a tablet such as the database and task upload/download with the goal to eliminate an external server entirely.
} 
- taskCredit records the number of tasks each device finishes and each user contributes.

- sendPush utilizes third party APIs to send notifications to registered devices. The Ocelot server (or dashboard) uses this function to notify clients that there are tasks available for computation.

- requestWork allows clients to request tasks to compute without receiving a push notification.

\subsection{Ocelot Client}

Ocelot, like BOINC, attempts to leverage public-resource computing but focuses on building middleware with energy efficiency and distribution flexibility. Although Ocelot is conceptually possible on many mobile platforms, our current implementation supports Android and iOS, shown in Figure 6 . Though the user interfaces (UIs) differ with platform, they share similar APIs for communicating with the servers and execute the same core algorithm. In the
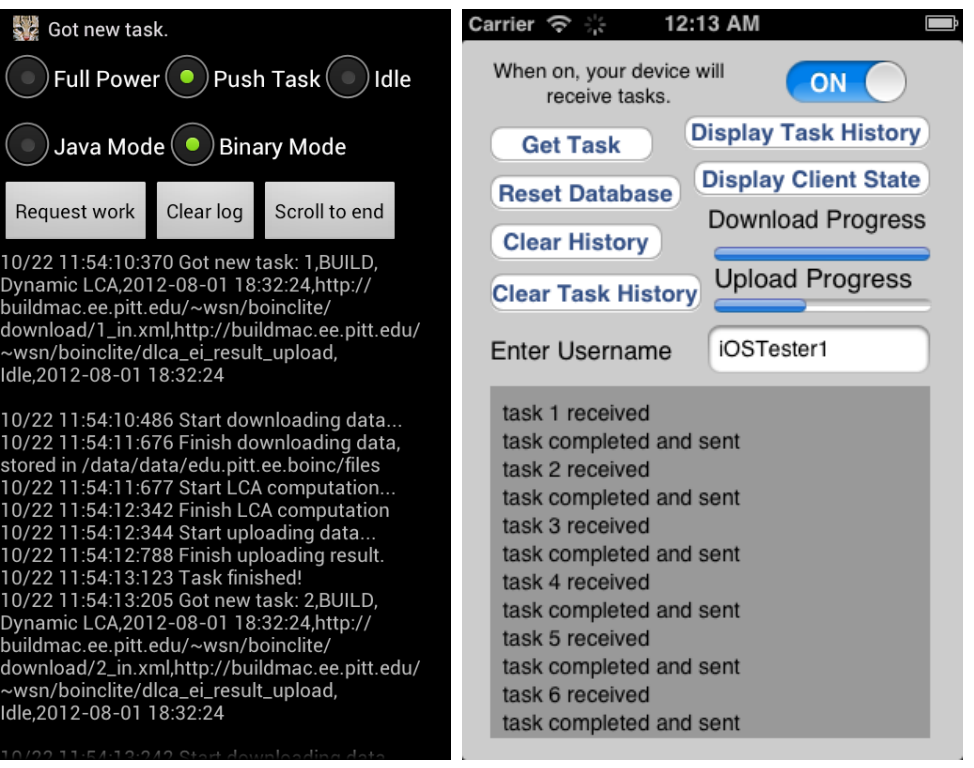

(a) Client on Android

(b) Client on iOS combined system of Ocelot and Lynx, all nodes share the same roles, comparable to peers in a P2P network. Because of this, a client can also become a server or even a dashboard, described in Section 4.4.

The limited battery capacities of mobile devices greatly restrict the amount of work that can be performed on a device operating as an Ocelot client. To provide the user some control over battery usage, the Ocelot client app is able to switch between different work modes defined by the user and client's power status. "Full Power" mode continuously requests tasks from the server, and is only available when set manually by the device's owner or when the device connects to an external power supply. In "Push Task" mode, the most commonly used mode, the client receives push notifications from the server and reacts according to the message content. The scheduling server notifies the clients when there are available tasks to be completed. For a client to receive push notifications requesting work, the client must be registered with the push server even if it has already installed the Ocelot client. The database server keeps a record of the power status of all clients (e.g. remaining battery life, charging status), by sending a periodic status fetch push to the client pool and letting the client report its power status after finishing each task. The scheduling server always selects clients that are connected to the power supply or have ample remaining battery life (e.g., above $50 \%$ in default settings). If no clients accept tasks after a preset period, devices with less power are notified. 
Those with very limited remaining power (e.g., below $20 \%$ in default settings) will not be pushed tasks. "Idle" mode is usually set by the device owner to avoid receiving pushed tasks from the Ocelot server. This mode allows the device to communicate on the network and potentially forward information from node to node, but otherwise conserves power.

A common Ocelot computation procedure begins with a task request from either a client or a dashboard. The client receives a task immediately after the request if there are unfinished tasks available. If the task request is initiated by a dashboard instead of a specific client, the scheduling server will push tasks to clients in non-idle mode according to their power status. Once a client receives a task, it examines whether the application code required is available locally on the device. If the client has never received a task from that application or if the application has been updated on the server, it downloads the application executable binary from the server and stores it locally for future use. Next, the working dataset is downloaded and the computation process begins, which could take seconds to minutes, depending on the task size. A code excerpt of binary execution on Android is shown in Listing 3. The Java method "Runtime.getRuntime().exec()" is used to run a Unix shell command at runtime on Android. We encourage smaller task sizes, which exploits parallelism, accelerating the computation and conserving individual batteries. The resulting file is uploaded to the server once computation completes and the client's latest power status is reported.

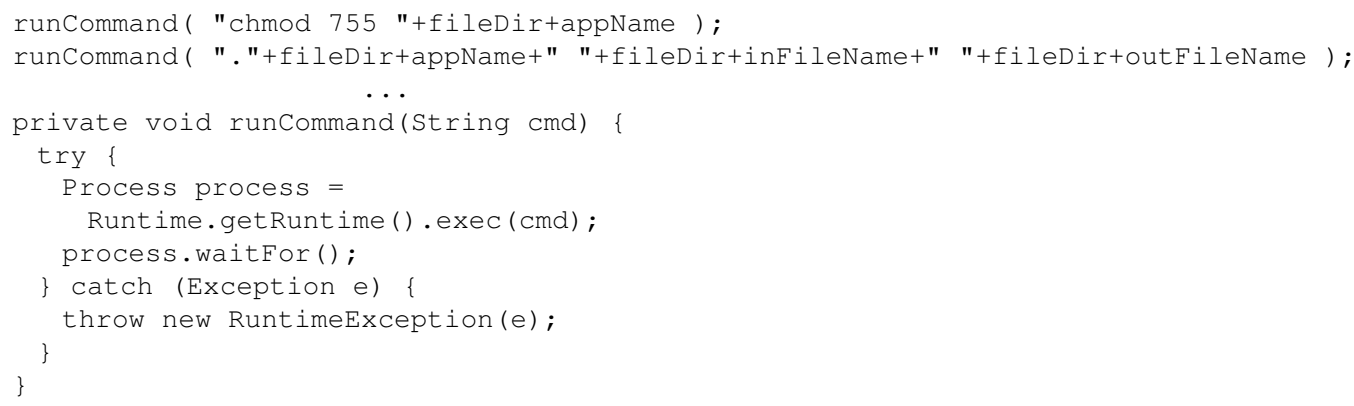

Listing 3: Binary execution on Android

\subsection{Ocelot Dashboard}

The Ocelot dashboard provides an on-demand, interactive charting engine to display result data from Ocelot computations. Figure 7 shows an interactive chart of one week of results for a life-cycle assessment algorithm performed on a building examining the energy consumption and environmental impacts over that period [21]. We describe this algorithm in more detail in Section 5.5. If a chart of results is requested for data that has not yet been computed, the dashboard will start the application on-demand to generate tasks to compute the requested display automatically. The dashboard user will be notified when computation is underway and when the chart is ready for display. Direct SQL query is supported on the Ocelot dashboard 
for advanced project administrators to easily modify databases, with potentially destructive actions such as deleting or truncating tables password protected.

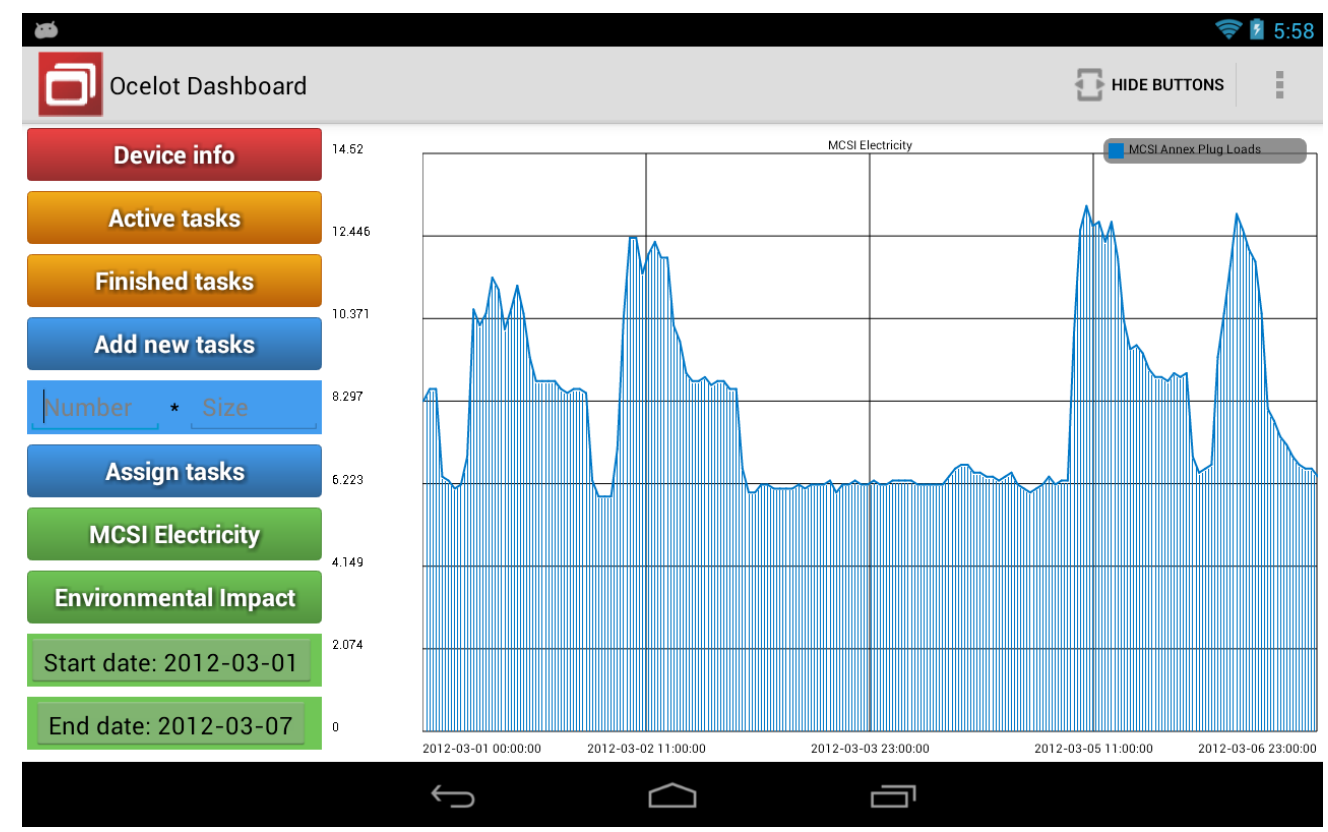

Figure 7: Ocelot dashboard

\subsection{Security and Limitations}

Security on mobile operating systems (OS) is a constant concern, as tablets often store personal information, and emerging new features of mobile devices provide additional opportunities for vulnerability. Both Android and iOS shield system files and resources from user apps, and restrict them from accessing files stored by other apps or from making changes to the device using a technique called "Sandboxing" [22]. Sandboxing prevents the Ocelot client app from malicious actions to the system or other apps and/or from being infected by other malicious apps. Since the App Sandbox is in the kernel, this security model extends to executing externally obtained native code and calling OS level applications. Moreover, other special mechanisms are enforced such as app permission requesting on Android and app code signing on iOS.

These safety mechanisms also create challenges in extending distributed computing to mobile devices. A direct effect is that binary code execution on iOS is prohibited. Rather than jailbreaking test devices, we combine the application code into the client app instead of downloading it from the server like the Android client. Thus, with new application codes, a new client app must be updated in iOS. We also maintain a record of the information of all participant devices by enforcing registration with Ocelot server before they can accept tasks. An owner's verified email addresses are associated with his/her device as the unique id, as an identifier in case of suspicious behavior. On Android devices, the owner's Google Play Store id or primary email account is used. However, iOS does not allow third-party apps to collect user information, so 
the user provides an id in the Client app. Similar limitations are subject to change in the future depending on the APIs released or updated by Google and Apple.

\section{Experimental Results}

To study the capabilities of Lynx we created a testbed of Android palmtop computers containing multiple Nexus 7 tablets. All were equipped with the Bluetooth 3.0 module and ran the Android 4.2.2 Operating System. Several heterogeneous setups of additional android devices were conducted. The results reported use homogeneous devices in order to provide an apples-to-apples comparison of the results of the multi-hop routing tests.

In order to ensure one-hop/multi-hop transfer, we manually configured connect daemon on all devices to set up the network into 1-hop, 2-hop and 3-hop structures with 4 different distances ranging from 1 to 15 meters, with the longest distance being separated by a wall. Due to the limited range of Bluetooth signal coverage, 15 meters was the greatest working distance (free of dropping connections) we could achieve. The tested data was a standard Ocelot task in XML format, with a size of 189,006 bytes (XML). A compressed version of the XML data, with a size of 22,610 bytes, was also tested (ZIP).

Each final value reported is an average of the results of six tests. The transfer time is calculated by the initiator device. The timer begins when the data transfer initates, and ends when a returned ACK signal is received. The averaged ACKing time was 348 milliseconds which is negligible compared to the transfer time.

\subsection{Data Transfer Time}

Figures 8(a) and 8(b) show transfer times of XML and ZIP, respectively. Not surprisingly, it took longer to transmit over longer distances. In addition, increasing the number of hops in the route nearly proportionally increases the transfer time, in most cases. The [2-hop, 5m, XML] (a total distance of $10 \mathrm{~m}$ ) scenario took nearly $50 \%$ longer than [1-hop, 10m, XML]. The [3-hop, 5m, XML] (a total distance of $15 \mathrm{~m}$ ) scenario took nearly $40 \%$ more time than [1-hop, 5m, XML], even with a wall in between. Routes with fewer hops and slower transmit rates usually outperform those with more hops and faster rates in most scenarios. However,

there is still a chance when a route with more fast links can outperform one with fewer slow ones, e.g., in the [2-hop, 5m, ZIP] scenario. Additionally, data compression reduces transfer time by $80 \%$.

When conducting the data transfer tests, we also measured the electrical source energy consumed by a device sending and receiving data, using Plugwise, a commercial metering system. Average sending power $P_{s}$ was $3.8 \mathrm{~W}$ and average receiving power $P_{r}$ was $2.2 \mathrm{~W}$, while simply running the app consumes $P_{o}$ of $1.9 \mathrm{~W}$. We also let the device calculate the total time $T_{z}$ needed for compressing the data, which was 579 milliseconds, on average. 


\subsection{Data Transfer Energy Consumption}

Figure 9 compares the energy consumption of transferring XML and ZIP in different scenarios side by side. The longer transmitting range requires more energy due to a slower rate, resulting in increased transfer time. And, similar to the transfer time discussed, energy consumption increases with the number of hops in most scenarios. Thus, the route with the least number of hops is typically more efficient than the route with shorter node-to-node distances. These results confirm that Lynx provides a system that can realistically model tradeoffs effectively for different system configurations.

An example of this can be shown in a comparison of compression overhead versus transmission overhead. Figure 10 compares energy savings in 12 different scenarios assuming a similar $88 \%$ data compression ratio. The results vary between $65 \%$ and $80 \%$, demonstrating that data compression in WSN communication greatly improves not only the network performance but, as expected, also improves the network lifetime by saving energy in distributed sensor nodes.

\subsection{Network Maintenance Energy}

As explained in Section 3.4, maintaining necessary peer and link states requires periodically running processes that also consume energy. We measured the average runtime of four processes including peer discovery, link establishment, and peer/link state report, and calculated the energy consumption based on the power measurement approach from Section 5.2. The "peer state report" has a similar packet size as the "link state report" so we combined the two energy components. The results are listed in Table 1. These overheads are all negligible compared to the data transfer energy.

\subsection{Network Scalability}


To investigate how Lynx performs with larger numbers of nodes and to quantify the impact of transient nodes, we increased the network size from four nodes to eight and 32 nodes. Figure 11 shows the average time of transferring a typical Ocelot input data unit of size 189,006 bytes. The average transfer time of each scenario is calculated based on results of 30 tests, through 1-, 2-, and 3-hop routes. We included situations where ad hoc nodes are forced to drop off the network, making the previously computed shortest paths invalid. There is a slight increase in transfer time due to these route invalidations, requiring nominal rerouting overheads. However, this overhead remains reasonable as the network scales.

\subsection{Performance of Ocelot Tasks on the} Lynx Network

Combining Lynx with Ocelot requires a distributed database and distributed task/resource management. For this approach, the SQLite database was employed for local node storage. Task and resource management is demonstrated through connection with a dashboard display. The dashboard node (e.g. a tablet)

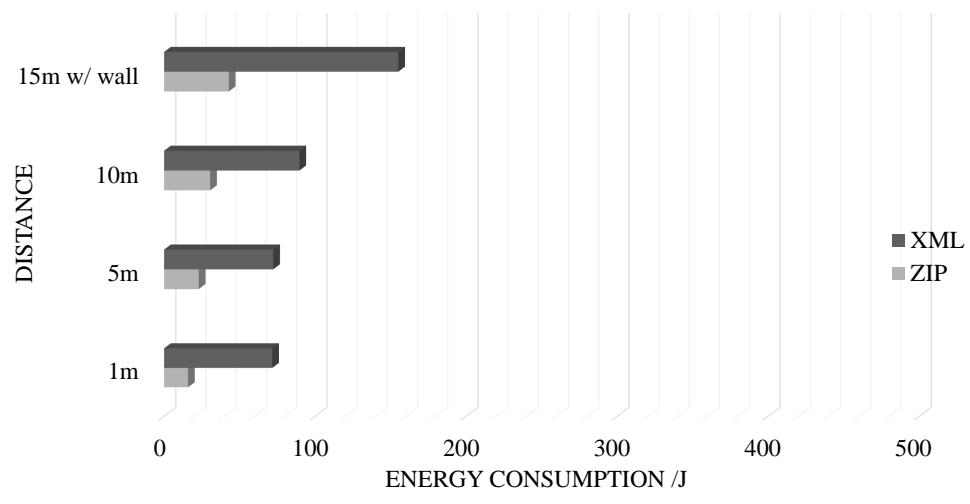

(a) 1-hop

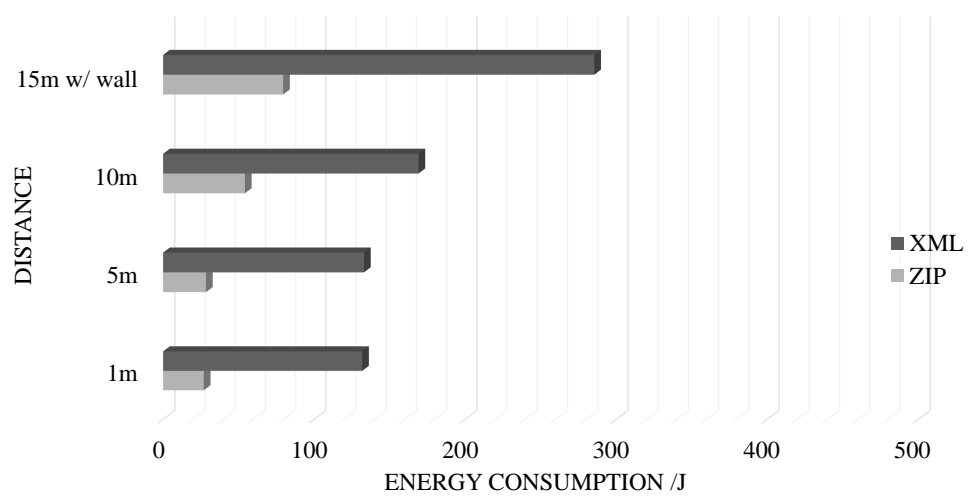

(b) 2-hop

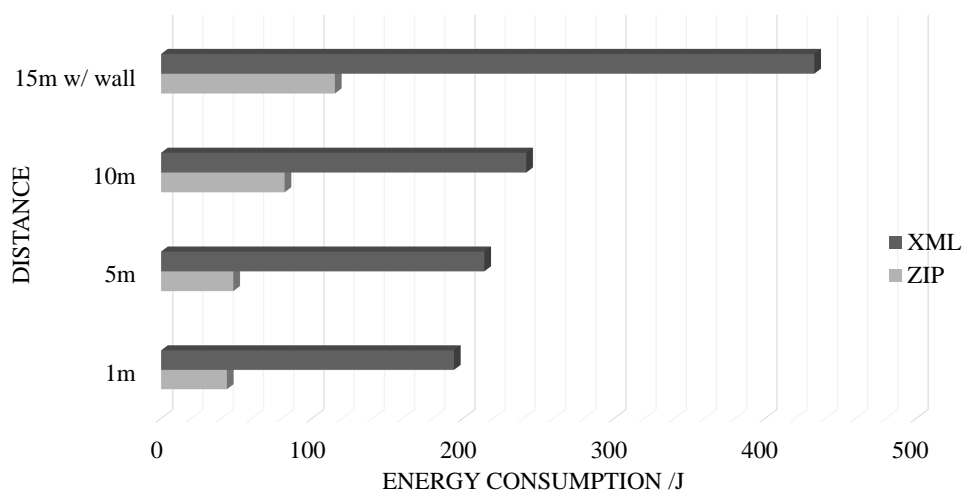

(c) 3-hop

Figure 9: Energy consumption of transferring uncompressed (XML) and compressed (ZIP) data.

Table 1: Energy Consumption of Miscellaneous Processes

\begin{tabular}{|c||c|c|c|c|}
\hline Daemon & $\begin{array}{c}\text { Peer } \\
\text { discovery }\end{array}$ & $\begin{array}{c}\text { Link } \\
\text { establishment }\end{array}$ & $\begin{array}{c}\text { Peer/link state } \\
\text { report (per hop) }\end{array}$ & $\begin{array}{c}\text { Route } \\
\text { calculation }\end{array}$ \\
\hline \hline Time /ms & 215 & 167 & 480 & 86 \\
\hline Energy /J & 0.41 & 0.50 & 1.44 & 0.16 \\
\hline
\end{tabular}


becomes both a Lynx and master Ocelot node. This node determines the data required to display a result for the user and automatically launches tasks for remote data collection and processing prior to aggregating the results for display. We conducted Lynx and Ocelot system experiments of task performance on the combined system. Similar to our previous tests, the network structures were different scenario combinations. The network locations of task input data were also configured to study the exact behaviors of communication in a controlled environment.

Several mobile devices as well as stationary computers were used in the experiments as listed in Table 2. As mentioned in Section 3, the current version of the Lynx app uses Bluetooth radio for communication. However, we have included the result of an experiment using WiFi-Direct as the data transfer method to show the potential of the latest $\mathrm{P} 2 \mathrm{P}$ con-

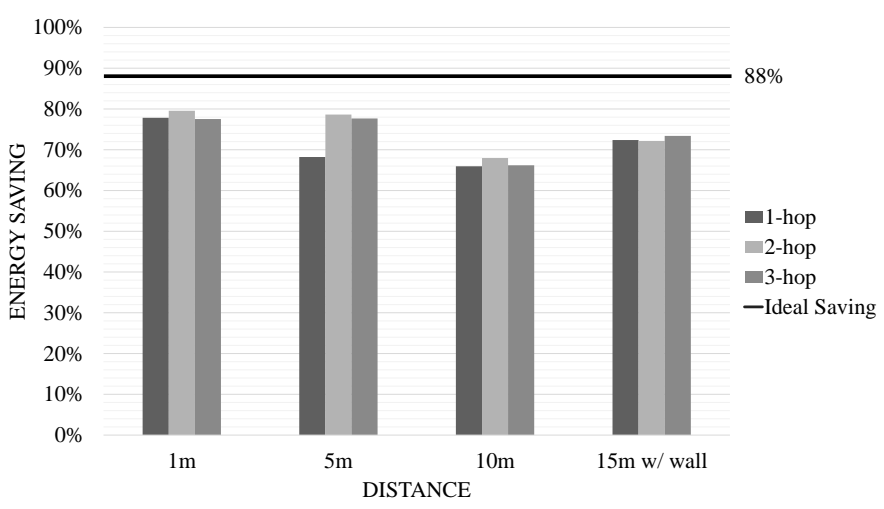
nectivity. Multi-hop routing over WiFi-Direct Figure 10: Energy savings by data compression in different scenarios is too inefficient due to hardware and API limitations, so only the 1-hop scenario was included here. And for simplicity, we only tested the five meter hop-to-hop distance. Performances in scenarios with other distances can be projected provided that the computation time is stable and the communication time was already presented in Section 5.1.

As a case study of the integrated system, we used dynamic life-cycle assessment (DLCA) on the electricity usage of the University of Pittsburgh's Mascaro Center for Sustainable Innovation (MCSI) building [8], and focused on the evaluation of electricity usage. Because electrical power is produced by power plants using various resources like coal, natural gas, nuclear fuel, etc., the production process itself consumes electricity as well. Our algo-

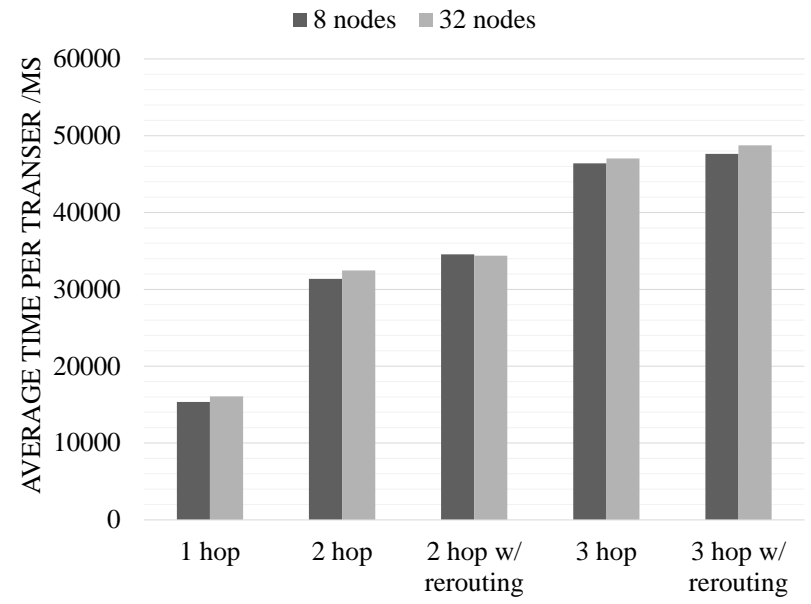

Figure 11: Average time of transferring 189,006 byte XML data, with different numbers of hops per route, and optional rerouting.

Table 2: Major specs of devices used in the experiments

\begin{tabular}{|c||c|c|c|}
\hline Device List & CPU & Memory & Power /w \\
\hline \hline Nexus 7 & 1.2G 4-core Cortex-A9 & 1GB & 2.2 \\
\hline \hline Mac mini (2010) & C2D P8600 & 8GB & 25.6 \\
\hline Mac mini (2011) & i5-2410M & 8GB & 23.9 \\
\hline Custom desktop 1 & Pentium G620 & 8GB & 57.2 \\
\hline Custom desktop 2 & Xeon 1230 v2 & 16GB & 127.0 \\
\hline Thinkpad W520 & i7-2760QM & 16GB & 32.1 \\
\hline
\end{tabular}


rithm calculates total electrical power needed to generate the electricity consumed in the building, as well as the environmental impacts it carries (e.g. all kinds of waterborne and airborne emissions). Additional historical data collected by some commercial meters is also used to provide workloads to Ocelot clients. For a DLCA within the building scope, electricity use is a significant component that demonstrates time sensitive variation, including by the hour of day and day of the week, and based on an exterior environment that changes in response to short term and seasonal weather cycles. Thus, these efforts have been heavily focused on better understanding electricity usage.

Figure 12 shows the average execution time per Ocelot task. Each task includes 1,000 data entries, each of which is similar to the structure of Listing 1. A total of 20 tasks were evaluated in each independent test. The distributed setups utilized the Lynx P2P network while the Ocelot-only systems [8] used an additional central server to obtain input data and send results back. The time for completing a task in the Lynx network was much longer due the slower Bluetooth protocol, especially when the number of hops in a route increases. Data compression helped to mitigate the file transfer time and as such, the total time. However, in the distributed WiFi-Direct test, the faster WiFi-Direct link also made the advantage of transferring smaller data units less evident. As a result, data compression failed to save time because of the overhead to compress and decompress the data.

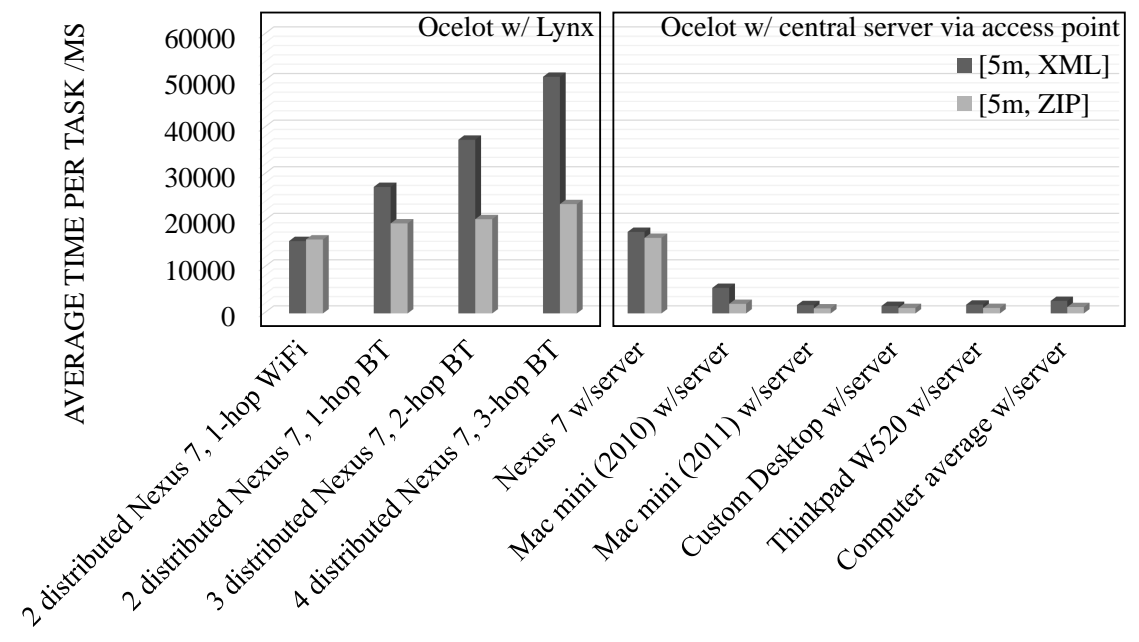

Figure 12: Average execution time per task

When considering Ocelot alone (i.e. computing nodes connected to a central server with a standard WiFi connection), traditional computers with more powerful processors outperform mobile devices. Lynx widened the gap due to slower transmission speeds. However, mobile devices consume less energy doing the same amount of work as shown in Figure 13. Without any powerful, yet power-hungry, central server, the fully distributed Ocelot system saved $70 \%$ energy even in the 3-hop BT scenario using data compression 
compared to the average computer consumption. Combining Ocelot with Lynx saved $88 \%$ energy with data compression and $78 \%$ without compression, according to the comparison of the [2 distributed Nexus7, 1-hop BT] scenario with the Ocelot-only [Nexus $7 \mathrm{w} /$ server] scenario. Moreover, full distribution of Ocelot using Lynx provides the best flexibility in constructing WSNs while also providing a computation facility, which is not typical of traditional WSNs or traditional distributed computing systems like BOINC.

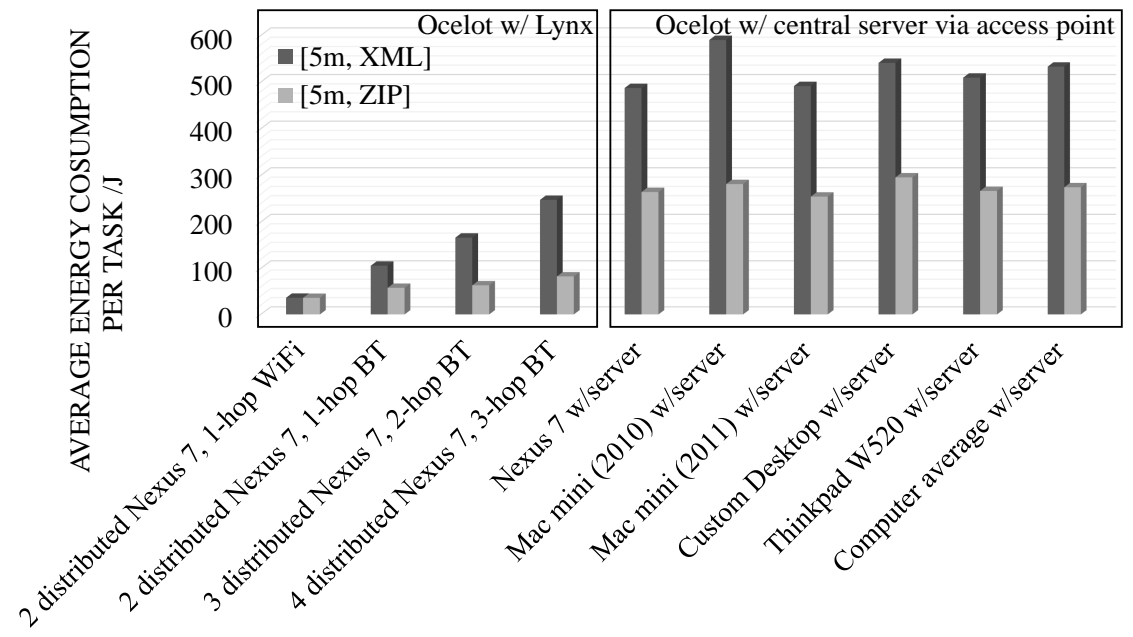

Figure 13: Average energy consumption per task

\section{Lynx as a testbed}

As introduced in Section 1, Lynx can serve as a natural platform for the study of various WSN routing and resource management algorithms, as the algorithm module is decoupled from the Lynx core, and is thus replaceable. The default algorithm used for multi-hop routing in Lynx is a modified version of Dijkastra's algorithm, as explained in Section 3.5. When the network is large enough such that there is more than one shortest path, the default algorithm arbitrarily selects one. However, a simple alternative is to choose the shortest path with the largest transmission rate to the next hop (Greedy algorithm). The detailed steps are described by Algorithm 2.

In this section, we demonstrate the capability of Lynx to serve as a testing platform of different routing algorithms in WSNs. We implement the greedy routing considering QoS (quality of service, in our case, transmission rate), and compare its performance to the default Dijkastra algorithm. A Lynx network of four nodes was set up as shown in Figure 14(a). Node 1 sends data to node 4 through two-hop routes with either 2 or 3 as the bridge node. Node 3 was placed closer to the sender $(\sim 1 \mathrm{~m})$ than node $2(\sim 10 \mathrm{~m})$, to achieve different (higher) transmission rate. A 189,006 byte XML packet was sent every 60 seconds and the transfer time of both routing algorithms was recorded and shown in Figure 15(a). The QoS algorithm always uses the 


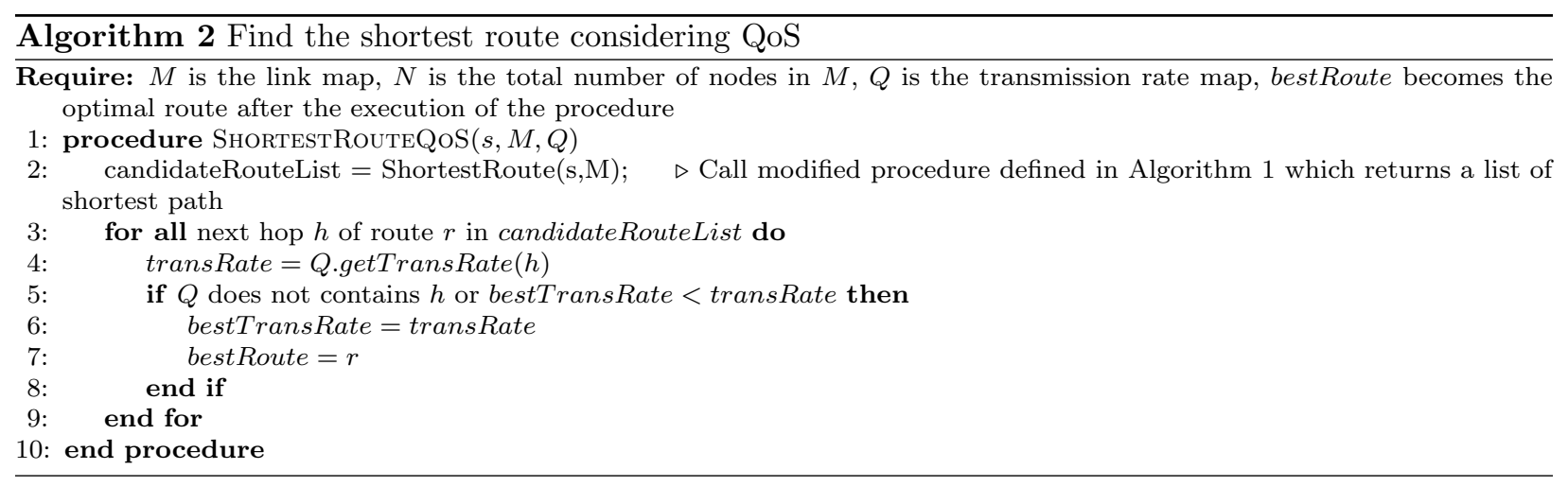

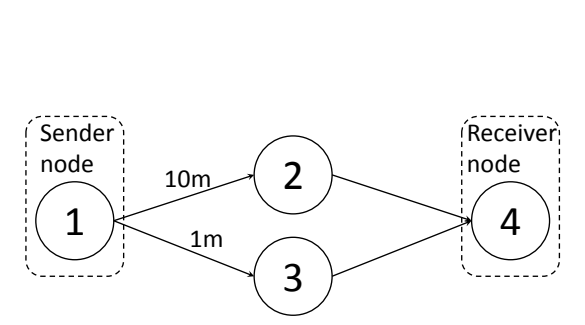

(a) 4-node network

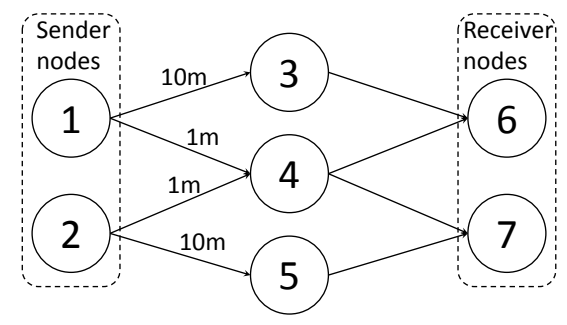

(b) 7-node network

Figure 14: Two network topologies used for testing the routing algorithm considering QoS. Edges indicate established connections; arrows indicate data transfer directions

faster route after having obtained the historical transmission rates of both routes, while the default Dijkstra routing arbitrarily chooses one.

Another experiment was carried out to find out the performance difference in a larger network (Figure 14(b)) with heavier traffic. The same XML data was sent from two sender nodes (1 and 2) to two receiver nodes (6 and 7) every 20 seconds instead of 60 seconds, and the average transfer time of the two concurrent transfers was recorded and shown in Figure 15(b). During the 20 second interval, data transfer completes the first hop of each route, but the second hop transfer is still in progress (given that the average 2-hop transfer of the same file takes about 30 seconds). Thus the newly initiated transfer is likely to interfere

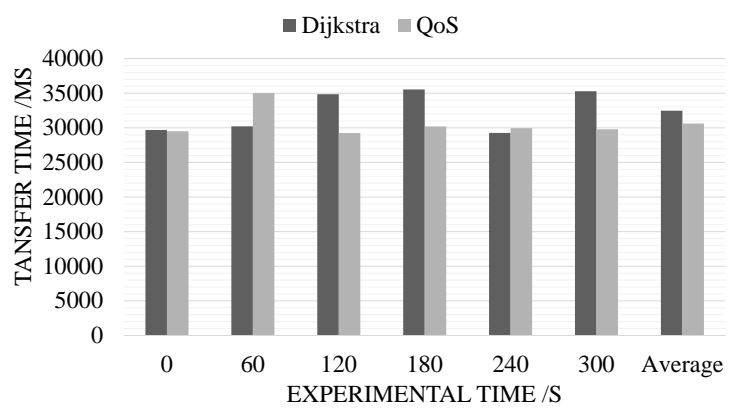

(a) 4-node network

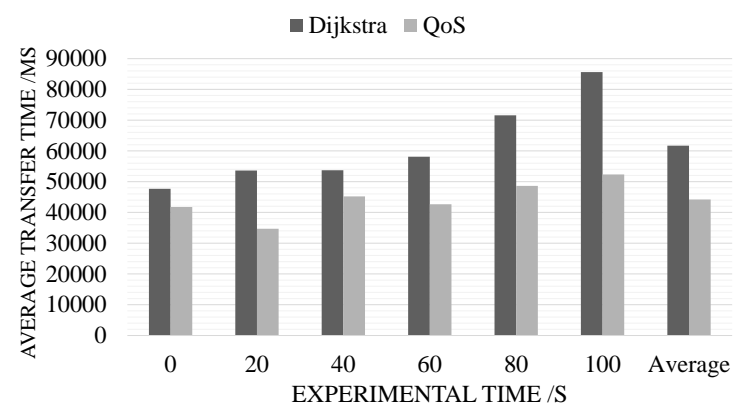

(b) 7-node network

Figure 15: Transfer time comparison of two routing algorithms. 
with the incomplete one and contend for bandwidth if two routes go through the same bridge node. The improved Dijkstra routing with QoS effectively avoids sustained performance degradation experienced with the original routing algorithm. In this case study, Lynx, serving as a testbed similar to software-based network simulators, effectively demonstrates the performance difference of different routing algorithms.

\section{Conclusion and Future Work}

The Lynx system provides a smart yet reliable approach to configure a wireless sensor network either for application in a system such as a smart building or as a testbed for WSN research. The self-organizing features are useful guides for us in designing and implementing WSNs in real world hardware, which reduces assumptions and inaccuracies in both impacts from different configurations and maintenance requirements.

As shown in the experimental results, WiFi-Direct connections provide a potentially useful WSN protocol from better speeds and the direct P2P links. Since the technology is less reliable, Lynx is possible with the Bluetooth protocol. Combining Lynx with Ocelot saves up to $88 \%$ energy compared to an Ocelot-only system, not to mention a more power-hungry grid platform such as BOINC. The flexibility that the LynxOcelot system provides in communication and distributed computing has great potential in application in areas ranging from health care and environmental data collection. The global network map maintenance and multi-hop routing modules laid the foundation for possible future directions such as real world testing of different routing algorithms.

\section{Acknowledgment}

This work was supported by National Science Foundation (NSF) under Grant EFRI-1038139.

\section{References}

[1] W. Guo, W. Zhang, A survey on intelligent routing protocols in wireless sensor networks, Journal of Network and Computer Applications 38 (2014) 185 - 201. doi:http://dx.doi.org/10.1016/j.jnca.2013.04.001.

[2] S. A. Nikolidakis, D. Kandris, D. D. Vergados, C. Douligeris, Energy efficient routing in wireless sensor networks through balanced clustering, Algorithms 6 (1) (2013) 29. doi:10.3390/a6010029.

[3] A. Pughat, V. Sharma, A survey on dynamic power management approach in wireless sensor networks, in: Proc. of PIICON, 2014, pp. 1-6. doi:10.1109/POWERI.2014.7117703.

[4] L. Paradis, Q. Han, A survey of fault management in wireless sensor networks, Journal of Network and Systems Management 15 (2) (2007) 171-190.

[5] G. Huang, Y. Zhang, J. He, J. Cao, Fault tolerance in data gathering wireless sensor networks, The Computer Journal 54 (6) (2011) 976-987. 
[6] TETCOS, Netsim, http://tetcos.com/netsim_gen.html (May 2014).

[7] Riverbed, Opnet, http://www.riverbed.com/products/performance-management-control/ opnet.html (May 2014).

[8] H. Xu, M. M. Bilec, L. A. Schaefer, A. E. Landis, A. K. Jones, Ocelot: A wireless sensor network and computing engine with commodity palmtop computers, in: Proc. of IGCC, 2013.

[9] D. P. Anderson, Boinc: A system for public-resource computing and storage, in: Proc. of GRID, 2004, pp. 4-10. doi:10.1109/GRID.2004.14.

[10] WiFi Alliance, Wifi-direct, http://www.wi-fi.org/discover-and-learn/wi-fi-direct (Jun. 2013).

[11] D. Camps-Mur, A. Garcia-Saavedra, P. Serrano, Device-to-device communications with wi-fi direct: overview and experimentation, IEEE Wireless Communications 20 (3) (2013) 96-104. doi:10.1109/MWC.2013.6549288.

[12] C. Gomez, J. Oller, J. Paradells, Overview and evaluation of bluetooth low energy: An emerging lowpower wireless technology, Sensors 12 (9) (2012) 11734-11753.

[13] Q. Yang, X. Zhu, H. Fu, X. Che, Survey of security technologies on wireless sensor networks, Journal of Sensors 2015 (842392) (2015) 9 pages. doi:10.1155/2015/842392.

[14] M. A. Sharaf, J. Beaver, A. Labrinidis, P. K. Chrysanthis, Tina: a scheme for temporal coherency-aware in-network aggregation, in: Proc. of Data engineering for wireless and mobile access, 2003, pp. 69-76.

[15] M. Abdul Azim, Z. Aung, S. Moad, N. Bouabdallah, M. E. Rivero-Angeles, I. Leyva-Mayorga, Energyefficient methods for highly correlated spatio-temporal environments in wireless sensor network communications, Wireless Sensor Network 2014 (6) (2014) 67-92. doi:doi: 10.4236/wsn.2014.65009.

[16] H. Xu, Y. Li, W. O. Collinge, L. A. Schaefer, M. M. Bilec, A. K. Jones, A. E. Landis, Improving efficiency of wireless sensor networks through lightweight in-memory compression, in: Proc. of IGSC, 2015, pp. 1-8. doi:10.1109/IGCC.2015.7393696.

[17] S. Feng, E. Seidel, Self-organizing networks (SON) in 3GPP long term evolution, Tech. rep. (2008).

[18] H. Hu, J. Zhang, X. Zheng, Y. Yang, P. Wu, Self-configuration and self-optimization for lte networks, Communications Magazine, IEEE 48 (2) (2010) 94-100.

[19] Qualcomm, Alljoyn, https://www.alljoyn.org/about (Jun. 2013).

[20] S. Verma, A. Robinson, P. Dutta, Audiodaq: turning the mobile phone's ubiquitous headset port into a universal data acquisition interface, in: Proc. of Embedded Network Sensor Systems, 2012, pp. 197-210.

[21] W. O. Collinge, L. Liao, H. Xu, C. L. Saunders, M. M. Bilec, A. E. Landis, A. K. Jones, L. A. Schaefer, Enabling dynamic life cycle assessment of buildings with wireless sensor networks, in: Proc. of ISSST, 2011, pp. 1-6.

[22] T. Blasing, L. Batyuk, A.-D. Schmidt, S. Camtepe, S. Albayrak, An android application sandbox system for suspicious software detection, in: Proc. of MALWARE, 2010, pp. 55-62. 


\section{Biographies}

Haifeng Xu received his Ph.D. from the University of Pittsburgh in 2015. His research interests include wireless sensor networks for sustainable systems and emerging non-volatile memory technologies. He is currently a Software Development Engineer at Amazon.

William Collinge is currently a Post-Doctoral Associate at the University of Pittsburgh. He received his Ph.D. from the University of Pittsburgh in 2013 and prior to that was a Project Engineer at AECOM for eight years. His research interests include life cycle assessment and green/high-performance buildings in the context of engineered systems and the built environment.

Laura Schaefer is the Burton J. and Ann M. McMurtry Professor and Department Chair of Mechanical Engineering at Rice University. She was a faculty member in Mechanical Engineering at the University of Pittsburgh from 2000-2015, where she was also a Bicentennial Board of Visitors Faculty Fellow and a Deputy Director of the Mascaro Center for Sustainable Innovation.

Amy Landis is the Thomas F. Hash Professor in Sustainable Development, Professor of Civil Engineering, and Director of the Institute of Sustainability at Clemson University. Previously, she was an Associate Professor in the School of Sustainable Engineering and the Built Environment at Arizona State University (2012-2015). She began her career as an Assistant Professor at the University of Pittsburgh (2007-2012).

Melissa Bilec is an associate professor in the Swanson School of Engineerings Department of Civil and Environmental Engineering; she is the Deputy Director of the Mascaro Center for Sustainable Innovation. Her research program focuses on the built environment, life cycle assessment, sustainable healthcare, and indoor air impacts.

Alex Jones is an Associate Professor of Electrical and Computer Engineering and Computer Science (by courtesy) at the University of Pittsburgh; he is the Director of the Computer Engineering program. His research program focuses on compilers, novel computer architectures, cyber-physical systems, and sustainable computing. 